\title{
The Ups and Downs of Bipolar Disorder Research
}

\author{
Richard S. Jope and Charles B. Nemeroff \\ Department of Psychiatry and Behavioral Sciences, Miller School of Medicine, University of \\ Miami, Miami, Florida.
}

\begin{abstract}
Bipolar disorder is a debilitating disease that continues to thwart a tremendous effort to understand its pathogenesis. Consequently, new treatment development has also languished. However, some inroads have been achieved. The first of these was undoubtedly the discovery that lithium stabilizes mood in a significant portion of patients with bipolar disorder. Not only was lithium pivotal for the treatment of this severe psychiatric disorder, but it also provided a critical tool to examine responses in patients and laboratory animals to acquire clues about the etiology of bipolar disorder (Figure 1), as indeed it was used in two reports published in this issue of Biological Psychiatry $(1,2)$. These reports each examined responses to lithium in applications of two newer advances applied to bipolar disorder, imaging technologies capable of examining human brains in vivo (3), and molecular methods that have identified genetic variations in subpopulations of patients with bipolar disorder (4). These reports exemplify the power by which bipolar disorder research can take advantage of the remarkable mood-stabilizing action of lithium in combination with newly developed technologies. However, just as the disease is characterized by unpredictable fluctuations in mood, so too are advances in understanding its etiology vulnerable to alternating cycles of apparent clarity and ambiguity.
\end{abstract}

Advances in structural and functional imaging of the human brain have provided unique insights in a multitude of neurologic and psychiatric disorders. Among these is bipolar disorder, in which imaging methods have helped to pinpoint affected brain regions and changes that occur in response to therapeutic interventions. One of the most intriguing and reproducible insights about bipolar disorder obtained from structural magnetic resonance imaging (MRI) studies are the reports (1) that lithium treatment increased brain gray area volumes in bipolar patients. This suggested that lithium may reverse degeneration or structural alterations of neurons that accompany the disease. That interpretation fit exceedingly well with evidence from preclinical studies that lithium increases adult neurogenesis (5), increases the production of neurotrophins, such as brain-derived neurotrophic factor, and reduces neuronal damage and death in response to a variety of insults (6), all mechanisms that could conceivably increase gray matter. However, the report by Cousins et al. (1) in this issue of Biological Psychiatry appears to have dashed the hopes

\section{(C) 2013 Society of Biological Psychiatry}

Address correspondence to Richard S. Jope, Ph.D., Miller School of Medicine, University of Miami, 1011 NW 15th St, Gautier Building, Room 416, Miami, Florida 33136; rjope@med.miami.edu.

CBN has received research support from the National Institutes of Health and the Agency for Healthcare Research and Quality. He has served as a consultant to Xhale, Takeda, and SKPharma. He has been a stockholder in CeNeRx Biopharma, NovaDel Pharma Inc, PharmaNeuroboost, Revaax Pharma, and Xhale. He has also had additional financial interests in Corcept, CeNeRx BioPharma, PharmaNeuroboost, Novadel Pharma, and Revaax. He has served on the scientific advisory boards of American Foundation for Suicide Prevention, AstraZeneca, CeNeRx Biopharma, Forest Labs, Janssen/Ortho-McNeil, Mt Cook Pharma Inc, NARSAD, NovaDel Pharma, Inc, Pharma-Neuroboost, Quintiles, and the Anxiety Disorders Association of America. He has also served on the Board of Directors for the American Foundation for Suicide Prevention, George West Mental Health Foundation, NovaDel Pharma, Inc., and Mt. Cook Pharma Inc. He holds a patent on the method and devices for transdermal delivery of lithium (US 6,375,990 B1) and the method to estimate drug therapy via transport inhibition of monoamine neurotransmitters by ex vivo assay (US 7,148,027B2). RSJ reports no biomedical financial interests or potential conflicts of interest. 
that an important therapeutic action of lithium had been identified. They tested the hypothesis that lithium may directly alter the MRI signals, a possibility noted but not followed-up by some previous investigators, which could lead to the spurious conclusion that lithium increased gray matter volume. MRI signal intensities used for volumetric analysis are commonly derived from the T1 relaxation properties of water. The presence of lithium shortens the T1 relaxation properties of water, which alters the MRI signal, tissue contrast, and volumetric analysis. Cousins et al. (1) showed that this direct effect of lithium on MRI signals can lead to the erroneous conclusion that lithium treatment increases gray matter volume, in part by showing that lithium did not alter gray matter volume when calculated using methods independent of T1 relaxation properties. This clarification answers the riddle of why MRI measurements indicated that gray matter volumes were increased by lithium administered to healthy controls who presumably did not suffer from abnormal neuronal degeneration or reduced neurogenesis, although, as the authors noted, the reported lithium-induced changes were generally greater in bipolar patients than control subjects. Will this finding end attempts to identify responses to lithium using MRI techniques? This is highly unlikely, and hopefully the opposite will occur, as the authors challenge other investigators to reexamine their data and to take into account lithium's direct effects on MRI signals in future studies. Such rigorous reassessments can be expected to lead to revisions in our understanding of how lithium affects the brain and to reveal new leads for future investigations. This report is an important reminder that defining the limitations of new technologies is as valuable as findings of significant changes. In the long term, this report should enhance the power of imaging techniques to clarify responses to lithium and other therapeutic agents.

Examining the effects of lithium administration also played an important role in the study by Leussis et al. (2) in this issue of Biological Psychiatry. They followed up the finding from genome-wide association studies (GWAS) that ankyrin 3 ( $A N K 3$ ) is a risk gene for bipolar disorder by studying ANK3-deficient mice. A broad range of analyses revealed alterations in only certain behaviors, particularly those commonly attributed to anxiety assessments, i.e., the elevated plus maze and light-dark transition measurements. In these behaviors, ANK3 deficiency reduced anxiety-like behaviors, which could be interpreted as being related to the mania-linked characteristic of increased risk taking, and these diminished anxiety-like responses of ANK3-deficient mice were normalized by lithium treatment. After being singly housed for 6 weeks, ANK3-deficient mice displayed the intriguing trait of responding to the isolation stress in a somewhat opposite manner to wild-type mice in anxiety-related behaviors, and isolation-stressed ANK3-deficient mice also displayed diminished preference for sucrose and increased despair, or depression-like behavior, in the forced swim test. Thus, the authors noted that chronic isolation stress induced a transition in ANK3-deficient mice "from decreased anxiety-related behaviors and increased motivation to depression-related features" (2) and suggested this was due to altered corticosterone regulation in ANK3deficient mice. Still to be determined are the effects of lithium on the behavioral responses to stress in the ANK3-deficient mice. These findings reveal a novel gene-environment interaction between ANK3 and stress. This is a valuable confirmation that GWAS, often criticized for failure in studies of psychiatric diseases, are providing meaningful clues about factors involved in bipolar disorder that can be studied in rodents, although the difficulties in interpreting rodent behaviors in terms of mood disorders continues to be an important limitation, as discussed previously (7). However, most importantly, this study can be considered as another step in the validation of the utility of GWAS in identifying risk genes for bipolar disorder and demonstrates the important role played by lithium in supporting links to bipolar disorder in behavioral measurements in rodents.

Both of these reports $(1,2)$ demonstrate the important role that advanced technologies play in biomedical research in general and bipolar disorder more specifically but also reveal the 
difficulties in interpreting results, and they highlight the continuing seminal position that lithium has in bipolar disorder research (Figure 1). Imaging and genetic advances provide the means to delve into aspects of bipolar disorder not possible a decade ago. But limitations in the manner in which the data are interpreted and related to bipolar disorder continue to obstruct advances. Computer-generated analyses that are required for handling the large databases obtained in imaging and genetic studies can generate many novel findings but also make difficult critical analyses of individual findings. The MRI findings of lithium-induced increased gray matter volumes fit so well with preclinical studies of lithium's effects that this may have helped to obscure technical problems that perhaps should have been evaluated more closely initially. Risk genes identified in GWAS can generate new hypotheses about bipolar disorder etiology, but testing these in rodents that may not be capable of displaying manic and depressive behaviors requires a stretch of interpretation of genetic links to bipolar disorder. However, we can still rely on lithium, the remarkable ion that remains the gold standard of treatment for patients with bipolar disorder and serves an invaluable role in bipolar disorder research. It is difficult to imagine examining bipolar disorder-linked phenotypes in rodent models without including tests of lithium's effects. Furthermore, identified responses to lithium finally can often be linked to mechanisms. A decade ago lithium appeared to have a multitude of targets, but these have been narrowed down because a great many of the actions of lithium now can be attributed to its inhibition of glycogen synthase kinase-3 (GSK3) (7). GSK3 is currently known to phosphorylate nearly 100 substrates (8), so its inhibition by lithium can account for many of the diverse actions of lithium that have been reported, including the activation of Akt and the extracellularly regulated kinase cascade, increased neurogenesis, and altered synaptic plasticity $(9,10)$ mentioned by Leussis et al. (2). Thus, clarification of the mechanism of action of lithium goes hand in hand with deciphering the causes of bipolar disorder, and lithium may prove to be as valuable in stabilizing fluctuations in bipolar disorder research as it is in stabilizing mood in bipolar disorder.

\section{Acknowledgments}

Research in the authors' laboratories was supported by grants from the National Institute of Mental Health (MH038752 and MH094759).

\section{References}

1. Cousins DA, Aribisala B, Ferrier IN, Blamire AM. Lithium, gray matter, and magnetic resonance imaging signal. Biol Psychiatry. 2013; 73:652-657. [PubMed: 23158114]

2. Leussis MP, Berry-Scott EM, Saito M, Jhuang H, de Haan G, Alkan O, et al. The ANK3 bipolar disorder gene regulates psychiatric-related behaviors that are modulated by lithium and stress. Biol Psychiatry. 2013; 73:683-690. [PubMed: 23237312]

3. Strakowski SM, Adler CM, Almeida J, Altshuler LL, Blumberg HP, Chang KD, et al. The functional neuroanatomy of bipolar disorder: a consensus model. Bipolar Disord. 2012; 14:313325. [PubMed: 22631617]

4. Lee KW, Woon PS, Teo YY, Sim K. Genome wide association studies (GWAS) and copy number variation $(\mathrm{CNV})$ studies of the major psychoses: what have we learnt? Neurosci Biobehav Rev. 2012; 36:556-571. [PubMed: 21946175]

5. Hanson ND, Nemeroff CB, Owens MJ. Lithium, but not fluoxetine or the corticotropin-releasing factor receptor 1 receptor antagonist R121919, increases cell proliferation in the adult dentate gyrus. J Pharmacol Exp Ther. 2011; 337:180-186. [PubMed: 21220416]

6. Beurel E, Jope RS. The paradoxical pro- and anti-apoptotic actions of GSK3 in the intrinsic and extrinsic apoptosis signaling pathways. Prog Neurobiol. 2006; 79:173-189. [PubMed: 16935409]

7. Jope RS. Glycogen synthase kinase- 3 in the etiology and treatment of mood disorders. Front Mol Neurosci. 2011; 4:16-26. [PubMed: 21886606] 
8. Sutherland C. What are the bona fide GSK3 substrates? Int J Alzheimers Dis. 2011; 2011:505607. [PubMed: 21629754]

9. Bradley CA, Peineau S, Taghibiglou C, Nicolas CS, Whitcomb DJ, Bortolotto ZA, et al. A pivotal role of GSK-3 in synaptic plasticity. Front Mol Neurosci. 2012; 5:13. [PubMed: 22363262]

10. O’Brien WT, Huang J, Buccafusca R, Garskof J, Valvezan AJ, Berry GT, Klein PS. Glycogen synthase kinase-3 is essential for $\beta$-arrestin- 2 complex formation and lithium-sensitive behaviors in mice. J Clin Invest. 2011; 121:3756-3762. [PubMed: 21821916] 


\section{Identification of lithium as a mood stabilizer}

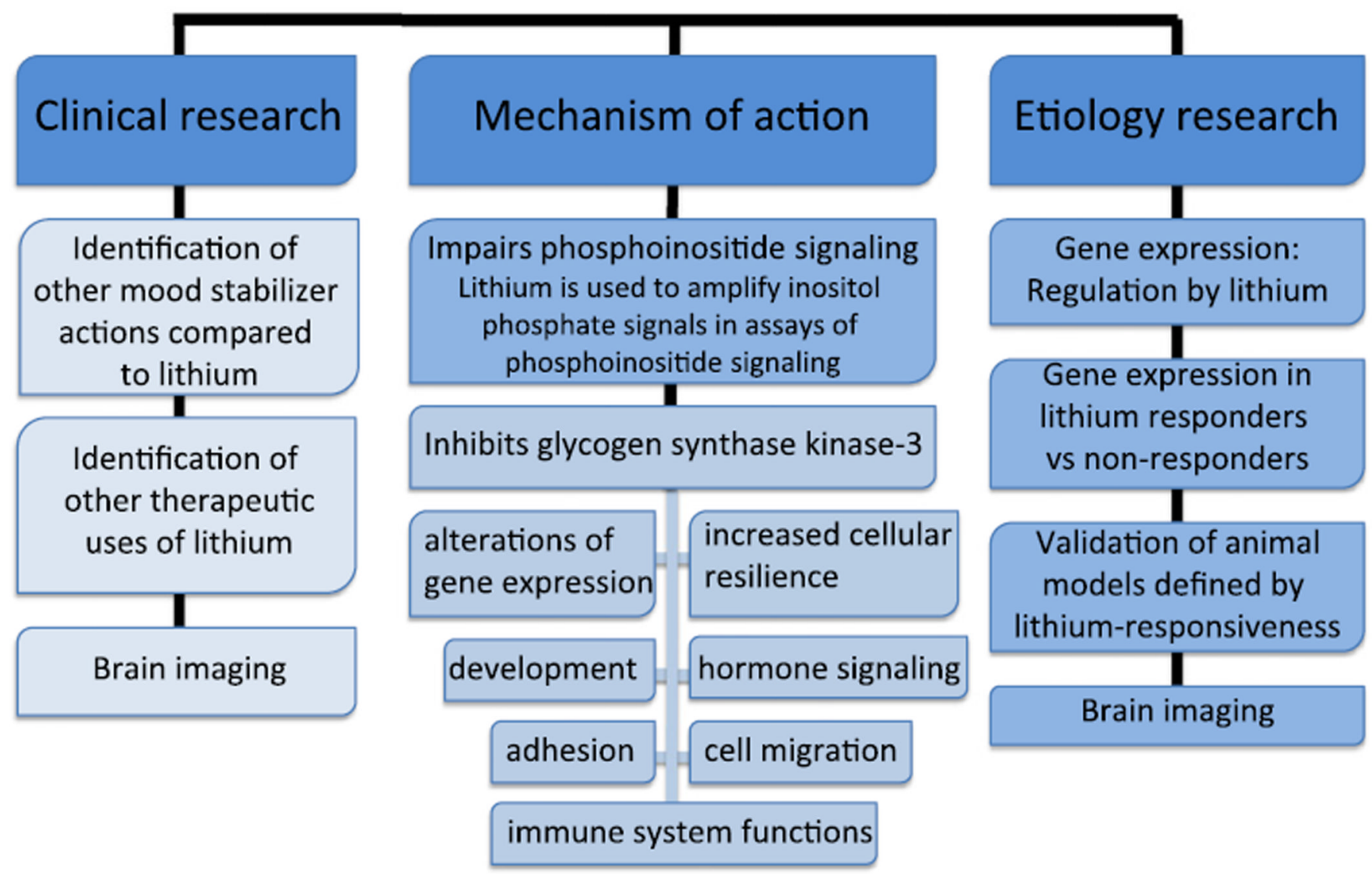

Figure 1.

Lithium has a central role in multiple aspects of bipolar disorder research. Diagram shows some of the many uses of lithium that have followed its identification as a mood stabilizer for bipolar disorder. These include clinical research efforts to identify new mood stabilizers, to identify other therapeutic applications of lithium, and to use imaging techniques to identify responses to lithium and other mood stabilizers. Lithium has also proven invaluable in basic research derived from studies of its mechanism of action. Lithium inhibits inositol phosphatases, and this led to the widespread use of lithium in the most common assays of the activity of the phosphoinositide signal transduction system. Lithium was the first identified inhibitor of glycogen synthase kinase-3, allowing studies to identify actions of this kinase, which is now known to phosphorylate nearly 100 substrates and to regulate numerous cellular actions. Lithium also has been a central figure in studies of the causes of mood disorders. Among these applications are studies of gene expression regulation by lithium, comparisons in patients with bipolar disorder distinguished by their therapeutic response to lithium, validation of animal models of mood disorders based on responses to lithium measured in behavioral assays, and uses of brain imaging to detect abnormalities that are corrected by lithium. 